\title{
TITLE:
}

\section{Turbulent cascade in vortex dynamics of magnetized pure electron plasmas}

$\operatorname{AUTHOR}(\mathrm{S}):$

Kawai, Y; Kiwamoto, Y; Soga, Y; Aoki, J

\section{CITATION:}

Kawai, Y ...[et al]. Turbulent cascade in vortex dynamics of magnetized pure electron plasmas. Physical Review E 2007, 75(6): 066404.

ISSUE DATE:

2007-06

URL:

http://hdl.handle.net/2433/50222

RIGHT:

Copyright 2007 American Physical Society 
PHYSICAL REVIEW E 75, 066404 (2007)

\title{
Turbulent cascade in vortex dynamics of magnetized pure electron plasmas
}

\author{
Y. Kawai, ${ }^{*}$ Y. Kiwamoto, Y. Soga, and J. Aoki \\ Graduate School of Human and Environmental Studies, Kyoto University, Yoshida Nihonmatsu-cho, Sakyo-ku, Kyoto 606-8501, Japan
}

(Received 8 April 2007; published 29 June 2007)

\begin{abstract}
Elementary processes in free-decaying two-dimensional (2D) turbulence are examined by extensive analyses of fine structures in the density distribution of a magnetized pure electron plasma that is linearly unstable with the initial ring-shaped profile, deforms nonlinearly into patches of vortices, and relaxes into a single-peaked stable distribution via successive mergers among the patches. The stochastic dynamics of the decreasing number of vortices in the real space correspond to the time evolution of energy spectra $E(k)$ in the wave number $(k)$ space that the energy transfers down to lower $k$ while the upward-spreading tails of the spectra fit to the power-law $\left(\propto k^{-\alpha}\right)$ with $\alpha>3$. The transfer rates, $\varepsilon(k)$ and $\eta(k)$ in energy and enstrophy spaces, evaluated from the time-resolved $k$-spectra demonstrate characteristic features of the $2 \mathrm{D}$ turbulence, i.e., $\varepsilon(k)$ is negative and deepest below the $k_{\text {inj }}$ corresponding to the size of the first-generated patches, and $\eta(k)$ increases from zero to a constant value at $k>k_{\text {inj. }}$. By averaging the time-dependent spectra of $E(k), \varepsilon(k)$, and $\eta(k)$, constructions are carried out for the spectra in a stationary turbulence that is sustained by the continuous generation of vortices due to the instability and dissipation at high $k$. The spectra are qualitatively consistent with the 2D turbulence theory with discrepancies including that $\alpha \approx 4.4$ and that the enstrophy transfer rate is almost zero around $k=k_{\text {inj }}$ reflecting the contribution from the coherent vortices.
\end{abstract}

DOI: 10.1103/PhysRevE.75.066404

PACS number(s): 52.27.Jt, 47.27.Jv

\section{INTRODUCTION}

Macroscopic dynamics of pure electron plasmas transverse to a strong magnetic field are equivalent to the twodimensional (2D) Euler fluid within the guiding-center approximation, and the electron density $n(x, y)$ and the potential distribution $\phi(x, y)$ are interpreted as the vorticity $\zeta(x, y)$ and the stream function $\psi(x, y)$, respectively [1]. Taking advantage of this equivalence, magnetized pure electron plasmas have been employed extensively for detailed examinations of many aspects of $2 \mathrm{D}$ vortical dynamics that constitute the elementary processes of $2 \mathrm{D}$ turbulence, such as the advection, merger, filamentation of vortex patches [1-3], and the formation of quasisteady states with ordered structures $[4,5]$ in the course of the relaxation from nonequilibrium initial states.

Vortex patches are spontaneously generated in the nonlinear stage of the diocotron instability of the ring-shaped electron density distribution with a strong radial shear of the azimuthal flow [6,7]. Free relaxations of the unstable system include stochastic dynamics of vortex patches. Timeresolved spectral analyses have been carried out along the relaxation of the turbulent states, focusing on the particle transport [8]. In this paper, we extend these examinations further to explore fundamental properties of 2D turbulence of the vorticity distribution in terms of the transport of the energy and enstrophy in the wave-number $(k)$ space.

Theoretical examinations of 2D turbulence have been carried out by Kraichnan [9] for the forced turbulence and independently by Batchelor [10] for the free-decaying turbulence. According to these studies it is expected that in the isotropic and homogeneous 2D turbulence, the enstrophy injected at the length-scale of $l_{\text {inj }}\left(\propto 1 / k_{\text {inj }}\right)$ cascades at a con-

\footnotetext{
*kwaiyosuke@h01a0395.mbox.media.kyoto-u.ac.jp
}

stant transfer-rate of $\eta$ down to a scale of dissipation $l_{d}$ $\left(\propto 1 / k_{d}\right)$ and dissipates at smaller scales by viscosity. This cascade picture of $2 \mathrm{D}$ turbulence leads to an energy spectrum characterized by the power-law $E(k) \propto k^{-3}$ in the inertial range of $k_{\text {inj }} \leq k \leq k_{d}$ in the wave-number space.

The cascade process of the enstrophy has been investigated experimentally by using thin layers of electrolytes [11,12] and soap films [13], and $k^{-3}$ scaling has been observed. However, in the electrolytic layer experiments, the coarse-graining of the flow distribution to compute the velocity field and numerical noises associated with the differentiation of the velocity field to determine the vorticity distribution make it difficult to ensure a high signal-to-noise $(\mathrm{S} / \mathrm{N})$ ratio enough to resolve the dissipative processes both in space and in amplitude. And in the soap film experiments, the reliance on the frozen-turbulence hypothesis for converting the data from a time series to spatial distribution makes it difficult to independently analyze the spatial and temporal structures of the flows. For these reasons, there remains some uncertainties in comparing the vortex dynamics observed in the real space in these experiments with the theoretical picture of 2D turbulence described in the spectral space.

In the experimental investigation with pure electron plasmas, the vorticity distribution can be determined directly in terms of the electron density distribution $n(x, y)$ down to small length scales comparable to the electron gyro-radii where the guiding center approximation breaks down. In addition, because the velocity field $v(x, y)$ is determined by the integration of $n(x, y)$, the relevant physical quantities are less vulnerable to numerical noises. With these advantages, in this paper, we observe and analyze the cascade process of the free-decaying 2D turbulence in wide length scales extending from the injection scale to the dissipative scale. Moreover, by considering this phenomena as an elementary process of forced 2D turbulence, we derive the spectrum in the stationary state through time-averaging the evolution of spectra and 
compare with the Kraichnan-Batchelor theory.

\section{EXPERIMENTAL METHOD}

The experiment is carried out using a pure electron plasma trapped in a Malmberg-trap with a uniform magnetic field $\left(B_{0}=0.048 \mathrm{~T}\right)$ and a square-well potential, which is in the same configuration as previously reported $[3,8,14]$. A set of cathodes selected from a cathode array are activated for pulsed injection of electrons into the trap. After the repetitive operation of injection-trapping cycles followed by the relaxation period of many strings of electrons, the distribution of trapped electrons relaxes to a bell-shaped profile that is axially homogeneous over the trap length of $L \sim 80 \mathrm{~mm}$. The typical height of the relaxed density and its radial width are $n \leq 1.3 \times 10^{13} \mathrm{~m}^{-3}$ and $r_{p} \sim 10 \mathrm{~mm}$, respectively. Since the self-field potential is the deepest around the axis, a slight reduction of the external potential barrier at the injection side lets these electrons escape selectively toward the cathode array. Various profiles of the ring distribution are generated by combining the depth of the escaping potential, the duration of the gating and the profile of the bell-shaped distribution. The fastest-growing mode of the diocotron instability critically depends on the aspect-ratio (radius/thickness) of the ring distribution. The number of the first-generated vortex patches is higher for larger aspect-ratios $[7,15]$. Therefore the enstrophy injection scale is controllable by varying the shape of ring profiles as the initial condition of the unstable system.

The collective dynamics proceed while the electrons are held in the trap. After a prescribed time of isolation in the trap, the electrons are dumped onto the conducting phosphor screen biased at $7.5 \mathrm{kV}$ for the acceleration. The luminosity distributions are recorded using a charge-coupled-device $(C C D)$ camera with $512 \times 512$ pixels and a dynamic range of 16 bits. This conducting screen also serves as an electron collector for the absolute determination of the total number of trapped electrons $N_{Q}$. A linear relationship between the integrated luminosity $N_{L}$ and $N_{Q}$ has been confirmed $[14,16]$. Therefore, the density distribution $n(x, y)$ is absolutely measurable. Using the equivalence to the $2 \mathrm{D}$ Euler fluids, the measured density distribution $n(x, y)$ is related to the vortic-
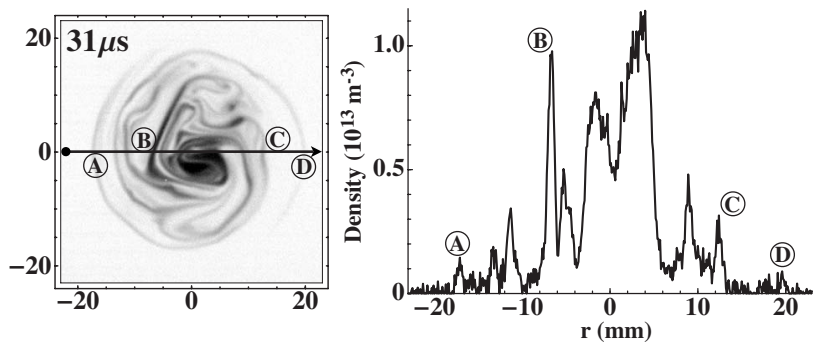

FIG. 1. An example of a measured 2D density distribution $n(x, y)$ (left) and a 1D profile along the chord $y=0$ (right). The positions labeled by the letters $\mathrm{A}$ to $\mathrm{E}$ on the $n(x, y)$ correspond to those of a cross-section profile.

ity distribution as $\zeta=e n / \varepsilon_{0} B_{0}$, and the potential distribution $\phi(x, y)$ obtained by solving the Poisson equation in combination with $n(x, y)$ is related to the stream function as $\psi$ $=\phi / B_{0}$, where $-e$ is the electron charge and $\varepsilon_{0}$ is the dielectric constant in vacuum. In this experimental configuration, the spatial resolution is $0.1 \mathrm{~mm} / \mathrm{pix}$ which is enough to resolve the dissipative scale $l_{d} \approx 0.32 \mathrm{~mm}$ as evaluated later. An example of the measured density distribution $n(x, y)$ and its profile along the chord $y=0$ are plotted in Fig. 1 to indicate that fine filamentary structures are clearly discernible.

The time evolution of the density distributions is observed by repeating the above procedure with varied times of the holding. Because the relaxation process of the turbulence triggered by the instability is stochastic in nature, a high reproducibility of the initial profiles is required for the destructive diagnostics. Therefore in this experiment, in addition to technically minimizing shot-by-shot variations in the initial profiles, an ensemble average is applied over typically 5 shots of data for each time of the observation in examining the time evolution of physical quantities characterizing the turbulence.

\section{RESULTS AND ANALYSES}

\section{A. Vortex dynamics in 2D turbulence}

The time evolution of the observed density distribution taken from one of the data sets is shown in Fig. 2. The ring
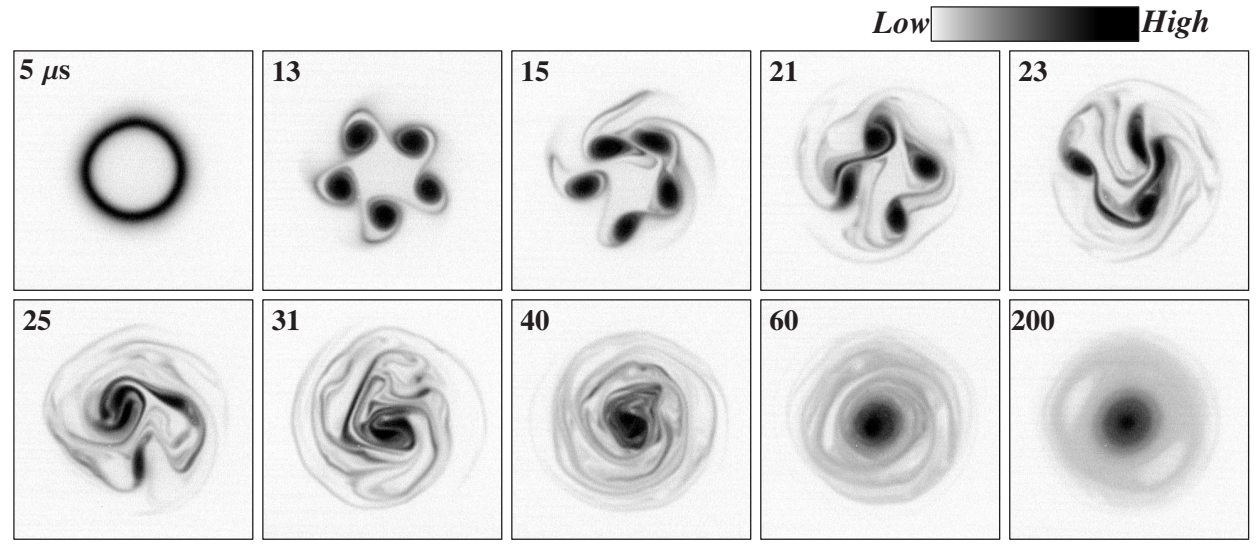

FIG. 2. Images of the time evolution of the density distribution. The darkness is proportional to the density. The time of observation (in $\mu \mathrm{s})$ is indicated at the upper left corner. 


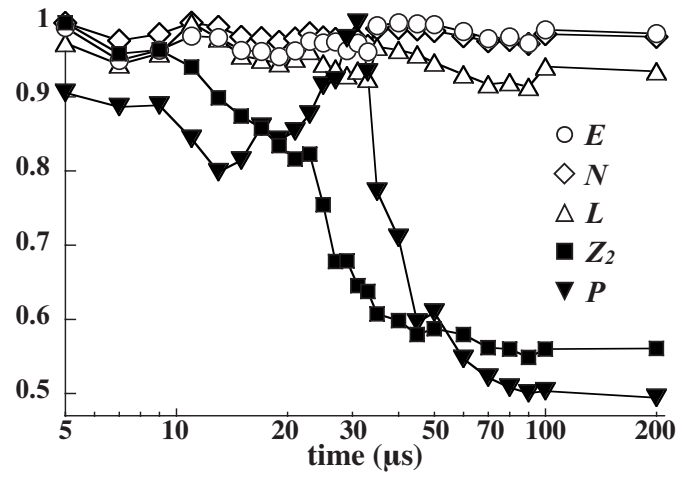

FIG. 3. The time evolution of energy $E$ (open circles), total electron number $N$ (open diamonds), angular momentum $L$ (open triangles), enstrophy $Z_{2}$ (solid squares), and palinstrophy $P$ (solid downward triangles).

profile is produced at $5 \mu \mathrm{s}$, and distorted by the diocotron instability within a few $\mu$ s. The deformed distribution grows on the fastest growing diocotron mode and eventually 5 high-density vortex patches are generated at $13 \mu \mathrm{s}$. After the formation of the first vortex patches, the number of patches decreases successively through intermittent mergers among the patches. This stochastic process is accompanied by the generation of filamentary structures in the low density part of the population that extend the length scales to finer regions. The outward transport of the filamentary structures is accompanied by the inward transport of the high-density patches as observed at $31 \mu \mathrm{s}$. The concentrated patches rotating with the period of $10 \mu$ s expel filaments from the central region, and finally form a bell-shaped core distribution that is surrounded by a low density halo with striations $(t=200 \mu \mathrm{s})$.

Turbulent states are characterized by areal integrals of the vorticity $[\propto n(x, y)]$. Figure 3 plots some of the low-order integrals calculated from the measured density distribution $n(r, \theta)$ as a function of time. Each integral is normalized to unity. The integrals include the electrostatic energy (fluid kinetic energy) $E=1 / 2 \int d^{2} \mathbf{r} n(-e \phi)$, the total electron number (total circulation) $N=\int d^{2} \mathbf{r} n$, the angular momentum (angular impulse) $L=\int d^{2} \mathbf{r} n r^{2}$, the enstrophy $Z_{2}=1 / 2 \int d^{2} \mathbf{r} n^{2}$, and the palinstrophy $P=1 / 2 \int d^{2} \mathbf{r}|\nabla n|^{2}$. Figure 4 shows the distribution of integrands in $Z_{2}\left[n(x, y)^{2}\right]$ and $P\left[|\nabla n(x, y)|^{2}\right]$ at $31 \mu \mathrm{s}$ in Fig. 2. The observation indicates that the enstrophy is concentrated at the high-density vortex patch and the palinstrophy is a measure of the fine-scale structures in the turbulence $[10,11,17]$.
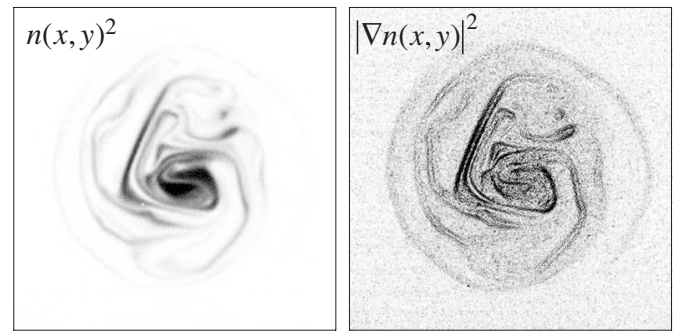

FIG. 4. The distribution of the integrand in $Z_{2}: n(x, y)^{2}$ (left) and in $P:|\nabla n(x, y)|^{2}$ (right) at $31 \mu$ s in Fig. 2.
Throughout the whole process, $E, N$, and $L$ do not show any systematic change except $5 \%$ variations probably attributable to the shot-by-shot fluctuations in the generation process of the unstable initial distributions. Therefore, the three integrals may be considered to be invariant. In contrast, $Z_{2}$ and $P$ show unambiguous systematic changes. The enstrophy $Z_{2}$ undergoes a substantial decaying through the merging processes, and finally goes down to $40 \%$ of the initial value. The palinstrophy $P$ shows a rapid increase while mergers among the vortex patches are active. It is maximized at $31 \mu$ s when the filamentary structures are conspicuous in the region outside the high-density core as shown in Fig. 2. After the maximization, $P$ drops steeply concomitantly with the reduction of the decreasing rate of the enstrophy, suggesting the manifestation of dissipative effects in finer length scales.

In order to estimate the degree of enstrophy dissipation in this experiment, we introduce a Newtonian viscosity into the $2 \mathrm{D}$ Euler equation as a correction. Because $\zeta=e n / \varepsilon_{0} B_{0}$, the Navier-Stokes equation is written as $D n / D t=\nu \nabla^{2} n$ (where $\nu$ is the kinematic viscosity). Multiplying $n$ to the equation and integrating it over the cross section under the boundary condition that $\left.n\right|_{r=R_{W}}=0$ (where $R_{W}$ is the wall radius of $32 \mathrm{~mm}$ ), the decreasing rate of the enstrophy is related to the viscosity and the palinstrophy as follows $[10,11,17]$ :

$$
\frac{D Z_{2}}{D t}=-2 \nu P
$$

By introducing the experimental values of $Z_{2}$ and $P$ in the interval from 13 to $31 \mu$ s into Eq. (1), the effective viscosity $\nu$ is evaluated as $0.004 \pm 0.002 \mathrm{~m}^{2} \mathrm{~s}^{-1}$.

The collisional transport of a magnetized pure electron plasma in the 2D regime has been studied analytically and experimentally [18]. In these studies, the viscosity coefficient is predicted theoretically as Eq. (62) in Ref. [19], and empirically from the experimental data as Eq. (12) in Ref. [20]. By introducing the parameters of the present experiment to the proposed formula, we estimate the viscosity coefficient as $0.004 \pm 0.003 \mathrm{~m}^{2} \mathrm{~s}^{-1}$ from Eq. (62) and as $0.0005 \pm 0.0003 \mathrm{~m}^{2} \mathrm{~s}^{-1}$ from Eq. (12). The former appears to be quantitatively consistent with the experimental evaluation based on Eq. (1). Though the present study is not under stationary states as assumed in the predictions, the agreement may suggest that the contribution of stochastic dynamics of individual particles under fluctuating fields is significant in the dissipation process of vortex dynamics in fine scales.

\section{B. Spectral dynamics in 2D turbulence}

To compare the experimental results to the theoretical picture of 2D turbulence, we calculate the energy spectrum in $k$ space from the measured density distribution. The energy spectrum $E(k)$ is determined from the Fourier transform of the density distribution $n(\mathbf{k})=\int d^{2} \mathbf{r} e^{-i \mathbf{k} \cdot \mathbf{r}} n(\mathbf{r})$ as

$$
E(k)=\frac{1}{2}\left(\frac{e}{\varepsilon_{0} B_{0}}\right)^{2} \int_{0}^{2 \pi} k d \varphi \frac{|n(\mathbf{k})|^{2}}{k^{2}},
$$

where $\varphi$ is the azimuthal angle of $\mathbf{k}$. The time evolution of $E(k)$ thus obtained is shown in Fig. 5. In the initial distribu- 


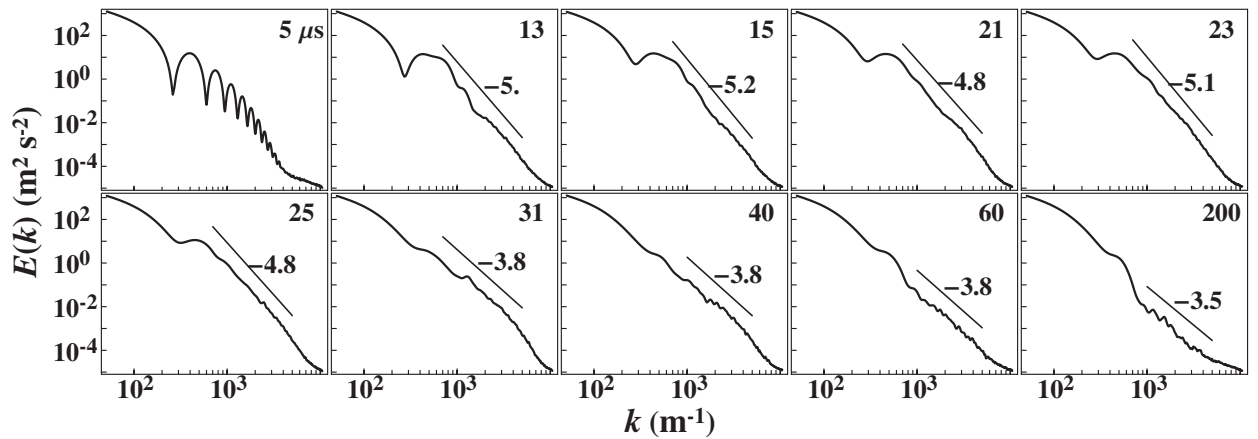

FIG. 5. The time evolution of the energy spectrum calculated from the measured $n(\mathbf{r})$. Numbers at the upper-left corner stand for the time of the observation.

tion at $5 \mu \mathrm{s}$, the energy spectrum shows oscillatory structures due to the concentration of the energy in the ring at the radius of $r_{p}$. When the ring distribution is torn into the vortex patches at $13 \mu \mathrm{s}$, the spectrum has a local maximum around the wave number $k=k_{\text {inj }} \approx 500$ consistent with the size of the first vortex patches. Along with the subsequent mergers between patches $(t=13-31 \mu \mathrm{s})$, the location of the spectral maximum shifts progressively down to lower wave numbers, and the dip around $k \equiv k_{\text {core }} \approx 300$ which corresponds to the core size in the final state is filled. In length scales smaller than the width of the vortex patches, the energy spectrum broadens upward and the $E(k)$ exhibits a power dependence of $\propto k^{-\alpha}$. The slope of the spectrum in the interval $700 \leq k$ $\leq 5000$ is drawn in Fig. 5 .

Throughout the merging processes, the power index $\alpha$ remains around 5, apparently larger than the theoretically predicted value of 3 [10]. In the slow relaxation process toward the asymptotic state after the merger $(t=40-200 \mu \mathrm{s})$, the energy concentrates at $k_{\text {core }}$ and decreases steeply beyond $k \approx 1000$. The power index in the high wave-number region $1000 \leq k \leq 5000$ decreases slowly from $\approx 5$ toward 3.5 . The power-dependent spectrum at high $k$ represents fine structures remaining in the halo region surrounding the high density core.

The rate of upward energy transfer $\varepsilon(k)$ through $k$ is evaluated from the time-resolved energy spectrum in Fig. 5 as

$$
\varepsilon(k)=-\int_{k_{\min }}^{k} d k \frac{\partial E(k)}{\partial t},
$$

where the lower integration limit $k_{\min }$ corresponds to the wall diameter. The enstrophy transfer rate $\eta(k)$ is evaluated simi- larly from the enstrophy spectrum $Z(k)=k^{2} E(k)$. The time evolution of $\varepsilon(k)$ and $\eta(k)$ during the period in which $E(k)$ follows the power law is shown in Fig. 6. The observation of $\varepsilon(k)$ indicates that the energy is transferred downward and the rate of transfers is maximum around $k_{\text {core }}$ at each time. In contrast, the enstrophy is transferred upward in the wavenumber space with $k \geq k_{\text {inj. }}$. Both in the energy and in the enstrophy, the transfer rates are maximized at $t \approx 25 \mu \mathrm{s}$ when the density configuration changes drastically by vortex merger from separated vortex patches to a single-peak distribution.

The experimental data indicate that, throughout the whole process, $\eta(k)$ is almost constant over the wide region of $k$ $\geq 1500$ as assumed in the theoretical picture of $2 \mathrm{D}$ turbulence. The rate is estimated at $\eta \approx(0.52-2.3) \times 10^{14} \mathrm{~s}^{-3}$ in Fig. 6. By combining the $\eta$ and the effective viscosity $\nu$ estimated in the previous subsection, the dissipative scale $l_{d}$ is estimated to be $0.32 \pm 0.07 \mathrm{~mm}$ according to the expression

$$
l_{d} \approx \eta^{-1 / 6} \nu^{1 / 2}
$$

proposed in Ref. [10]. This length is consistent with the thickness of the filamentary structure at the end of spiral arms displayed at the position D in Fig. 1. The observation that $\eta(k)$ decreases to zero at $k<2 k_{\text {inj }}$ from a constant value at $k>3 k_{\text {inj }}$ corresponds to the observed vortex dynamics in Fig. 2 showing that the change of the structure size is limited to the tenuous region outside the high-vorticity patches. The constraint of the enstrophy cascade by the vortex patches is considered as a reason why the slope of the observed energy spectrum is steeper than the theoretical prediction $[21,22]$.

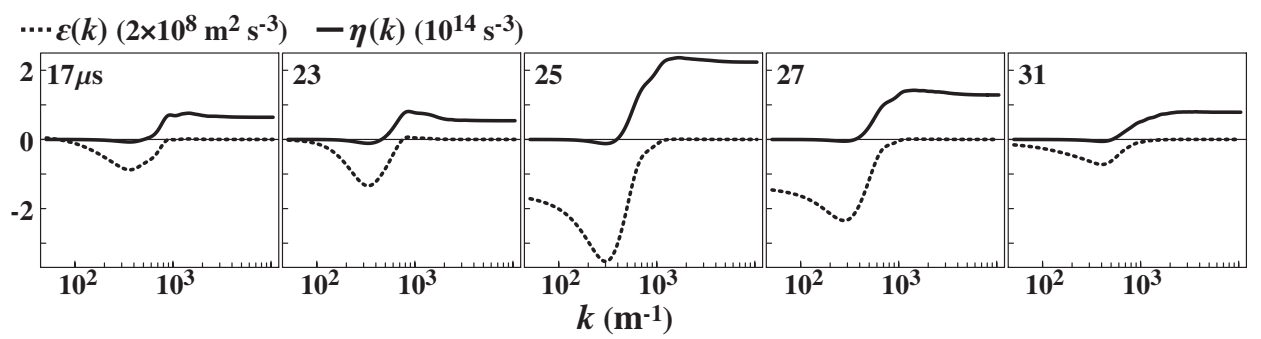

FIG. 6. The time evolution of the upward transfer rates of the energy $\varepsilon(k)$ (dashed line) and enstrophy $\eta(k)$ (solid line) through $k$. 


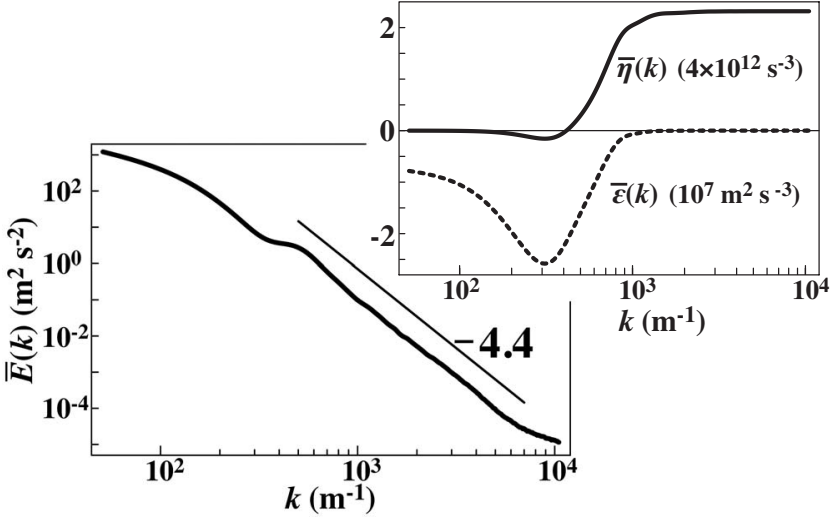

FIG. 7. The energy spectrum $\bar{E}(k)$ time-averaged in the interval $t=13-200 \mu \mathrm{s}$. Inset shows the time-averaged transfer rates of the energy $\bar{\varepsilon}(k)$ (dashed line) and enstrophy $\bar{\eta}(k)$ (solid line).

\section{Spectra in stationary turbulence}

The time-resolved data presented above include details of free-decaying 2D turbulence whose enstrophy is fed at $k$ $=k_{\text {inj }}$ corresponding to the first generation of vortex patches and that is left in an isolated state. Here we try to construct the energy spectrum in a stationary state that is maintained by continuous energy input at $k=k_{\text {inj }}$ due to the instability and by the continuous dissipation at $k \gg k_{\text {inj }}$ by using the above data as an elementary process constituting a stationary turbulence. If the interaction among the structures appearing in different stages of the free-decaying turbulence is negligible, the time-averaged spectra, $\bar{E}(k), \bar{\varepsilon}(k)$, and $\bar{\eta}(k)$, may approximately represent the characteristic features of the stationary turbulence. The results of the time-weighted average of the observed data are summarized in Fig. 7.

The constructed spectra exhibit characteristics close to the fundamental features of stationary 2D turbulence: The enstrophy is transferred upward at a constant rate above $k$ $\approx 1500=3 k_{\text {inj }}$ corresponding to one-third of the scale of first vortex patches. With this transfer rate, the dissipative wave number is estimated at $k_{d} \approx 7200$ from Eq. (4). Figure 7 also shows that $\bar{E}(k)$ depends algebraically on $k$ in a wide range of the wave-number space $k_{\text {inj }} \leq k \leq k_{d}$. In contrast to $\bar{\eta}(k)$, it is confirmed that in the region $k<3 k_{\text {inj }}$, the energy flux $\bar{\varepsilon}(k)$ proceeds toward small wave numbers and is maximized around $k_{\text {core }}$ corresponding to the size of the core distribution in the asymptotic state.

Through the region $k \geq 3 k_{\text {inj }}$, where the enstrophy transfer rate is constant, the energy transfer rate is observed to be zero. This observation supports the theoretical expectations that the spectral dynamics at high wave numbers of the $2 \mathrm{D}$ turbulence are governed by the enstrophy cascade process [9]. On the other hand, in the region $k<k_{\text {inj }}$, the observation that $\bar{\eta}(k) \sim 0$ and $\bar{\varepsilon}(k)<0$ indicates that the dynamics in the energy spectra obey the inverse cascade process. The observed nonuniformity of $\bar{\varepsilon}(k)$ is understood in terms of the absence of a dissipation mechanism at large length scales in the strongly magnetized pure electron plasma.

In the intermediate region $k_{\text {inj }} \leq k<3 k_{\text {inj }}$, neither $\bar{\varepsilon}(k)$ nor $\bar{\eta}(k)$ is zero, indicating that the transfer exists in the $k$ space of both the energy and enstrophy. The local nonuniformity of $\bar{\varepsilon}(k)$ and $\bar{\eta}(k)$ in this region suggests the breakdown of the ubiqitous cascade model. This observation is closely related to the persisting presence of coherent vortices that capture a large amount of enstrophy as shown in Fig. 4 and inhibit the cascade as observed by numerical simulations $[21,22]$. This is probably the reason why the power index of the observed energy spectrum $\bar{E}(k)$ in the inertial range of $k_{i n j} \leq k \leq k_{d}$ is $\alpha=4.4$ and larger than the theoretical prediction of $\alpha=3$ [9].

\section{CONCLUSION}

In this paper, we have examined the vortex dynamics of 2D turbulence in a magnetized pure electron plasma over a wide range of length scales extending from the injection scale down to the dissipative scale, and compared the experimental results to existing theories of 2D turbulence. In the stage characterized by the successive mergers between vortex patches starting from the unstable initial density profile, the observed density distribution exhibits turbulent characteristics. While the energy is transferred downward, the enstrophy undergoes an upward transport starting from the injec-

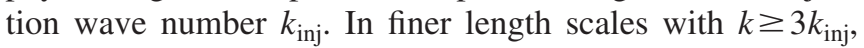
the transfer rate of the enstrophy is observed to be constant, and the energy spectrum shows a power-law scaling $E(k)$ $\propto k^{-\alpha}$ in a broad inertial range with $\alpha$ ranging from 5.2 to 3.5. The discrepancy from the theoretically expected value of $\alpha$ $=3$ is attributed to the inhibition of the cascade process reflecting the effect of the long persistence of high-vorticity patches.

\section{ACKNOWLEDGMENTS}

The author thanks Professor K. Itoh of NIFS for enlightening discussions. This research was supported by the Grantin-Aid for Scientific Research (B) 17340173 of JSPS and partly by the collaborative program of NIFS.
[1] C. F. Driscoll and K. S. Fine, Phys. Fluids B 2, 1359 (1990).

[2] T. B. Mitchell and C. F. Driscoll, Phys. Fluids 8, 1828 (1996).

[3] Y. Kiwamoto, K. Ito, A. Sanpei, and A. Mohri, Phys. Rev. Lett. 85, 3173 (2000).

[4] X.-P. Huang and C. F. Driscoll, Phys. Rev. Lett. 72, 2187 (1994).
[5] K. S. Fine, A. C. Cass, W. G. Flynn, and C. F. Driscoll, Phys. Rev. Lett. 75, 3277 (1995).

[6] R. H. Levy, Phys. Fluids 8, 1288 (1965).

[7] A. J. Peurrung and J. Fajans, Phys. Fluids A 5, 493 (1993).

[8] Y. Kawai, Y. Kiwamoto, K. Ito, A. Sanpei, Y. Soga, J. Aoki, and K. Itoh, J. Phys. Soc. Jpn. 75, 104502 (2006). 
[9] R. H. Kraichnan, Phys. Fluids 10, 1417 (1967).

[10] G. K. Batchelor, Phys. Fluids 12 (Suppl. II), 233 (1969).

[11] P. Tabeling, Phys. Rep. 362, 1 (2002).

[12] J. Paret, M. C. Jullien, and P. Tabeling, Phys. Rev. Lett. 83, 3418 (1999).

[13] M. A. Rutgers, Phys. Rev. Lett. 81, 2244 (1998).

[14] K. Ito, Y. Kiwamoto, and A. Sanpei, Jpn. J. Appl. Phys., Part 1 40, 2558 (2001).

[15] R. C. Davidson and G. M. Felice, Phys. Plasmas 5, 3497 (1998).

[16] J. Aoki, Y. Kiwamoto, Y. Soga, and A. Sanpei, Jpn. J. Appl. Phys., Part 1 43, 7267 (2004).
[17] U. Frisch, Turbulence (Cambridge University Press, Cambridge, UK, 1995).

[18] C. F. Driscoll, F. Anderegg, D. H. E. Dubin, D.-Z. Jin, J. M. Kriesel, E. M. Hollmann, and T. M. O’Neil, Phys. Plasmas 9, 1905 (2002).

[19] D. H. E. Dubin and T. M. O’Neil, Phys. Plasmas 5, 1305 (1998).

[20] J. M. Kriesel and C. F. Driscoll, Phys. Rev. Lett. 87, 135003 (2001).

[21] J. C. McWilliams, J. Fluid Mech. 146, 21 (1984).

[22] P. Santangelo, R. Benzi, and B. Legras, Phys. Fluids A 1, 1027 (1989). 